\title{
Complexities, Error Probabilities, and Capacities of Optical OOK-CDMA Communication Systems
}

\author{
Hossam M. H. Shalaby, Senior Member, IEEE
}

\begin{abstract}
The complexity in the hardware implementation of traditional optical code-division multiple-access correlation receivers with double optical hardlimiters is discussed. A comparison with the implementation of recently proposed chip-level receivers is presented as well. In addition, the bit error probabilities and the throughput capacities for both chip-level and correlation systems (without hardlimiters) are derived and evaluated under code-correlation constraints equal to one and two. Our results reveal that chip-level receivers are much simpler and their performances are competitive with that of traditional correlation receivers with double optical hardlimiters. Further, the throughput capacity of chip-level systems can be increased by almost a factor of 3.4 when increasing the code-correlation constraint from one to two.
\end{abstract}

Index Terms-Chip-level receivers, code-division multiple access (CDMA), correlation receivers, direct detection optical channel, on-off keying (OOK), optical CDMA, optical channel capacity, optical hardlimiters.

\section{INTRODUCTION}

B OTH OPTICAL code- and time-division multiple-access (CDMA and TDMA) techniques can be utilized in fiberoptic local and wide-area networks (LANs and WANs) because of the prodigious bandwidth offered by the optical links and the extra-high optical signal processing speed bestowed by the optical components. Consequently, it becomes possible to endow an immense amount of transmission capacity economically and accommodate a large number of simultaneous users in optical LANs and WANs. When compared to TDMA, optical CDMA techniques do not require time synchronization among different users, yet provide flexibility in the network design, security against interception, and complete utilization of the entire timefrequency domain by each subscriber [1]-[11]. Optical CDMA systems, on the other hand, suffer from multiple-user interference, which degrades their performance as the number of users increases. Further, they exhibit error probability floors, which cannot be reduced without the addition of interference cancellation subsystems [9].

The traditional method to recover the data at the receiving end of an optical CDMA system is to use an optical correlator followed by a photodetector and a decision device [2].

Paper approved by I. Andonovic, the Editor for Optical Networks and Devices of the IEEE Communications Society. Manuscript received May 10, 2000; revised February 20, 2002.

The author is with the Department of Electrical Engineering, Faculty of Engineering, University of Alexandria, Alexandria 21544, Egypt (e-mail shalaby@ieee.org).

Digital Object Identifier 10.1109/TCOMM.2002.806502
To enhance the performance of the correlation receiver, Salehi and Brackett used an optical hardlimiter before the correlator at the receiver side [2]. Although the performance of their receiver (which involved an ideal photodetector) was improved, Kwon [4] has shown that such improvement becomes insignificant for more realistic systems, e.g., with avalanche photodiodes (APDs). With the implementation of double optical hardlimiters before and after the correlator at the receiving end, Ohtsuki [6], [8] was able to significantly improve the error-rate performance.

Although many authors have adopted the optical hardlimiters in their systems' design, the problem with these devices is that their technology is not yet mature and their ideal characteristics are very difficult to implement [12], [13]. Recently, we have proposed a new receiver model, called a chip-level receiver [7]. This receiver does not require the optical hardlimiters or the correlators in its implementation, hence, it is much more practical than the correlation receiver with hardlimiters.

Our goal in this paper is twofold. First, we aim at comparing the chip-level receiver and the double-optical-hardlimiters correlation receiver in terms of hardware complexity and bit error probability. We employ the optical orthogonal codes (OOCs) [1], with periodic cross correlations and out-of-phase periodic autocorrelations that are bounded by one $(\lambda=1)$, as the users' signature code sequences in our theoretical analysis. This constraint on the code correlations guarantees minimal interference between the users at the expense of limiting the maximum number of subscribers. To increase the possible number of subscribers, we need to relax a bit the constraint on the code correlations. Our second aim in this paper is to evaluate the throughput and efficiency capacities of both optical on-off keying (OOK)-CDMA chip-level and correlation systems when using two different code-correlation constraints, namely, $\lambda \in\{1,2\}$.

The remainder of this paper is organized as follows. The optical OOK-CDMA receiver models are described in Section II and their hardware complexities are discussed. The bit error probabilities for the optical OOK-CDMA systems, under a code-correlation constraint equal to one, are compared in Section III, taking into account the effect of both the APD noise and the thermal noise, in addition to the multiple-user interference. Section IV is devoted to the development of the bit error probabilities for optical OOK-CDMA chip-level receivers with a code-correlation constraint equal to two. In Section V, we compare the throughput and efficiency capacities of OOK-CDMA systems under different code-correlation constraints. Finally, the conclusion is given in Section VI. 


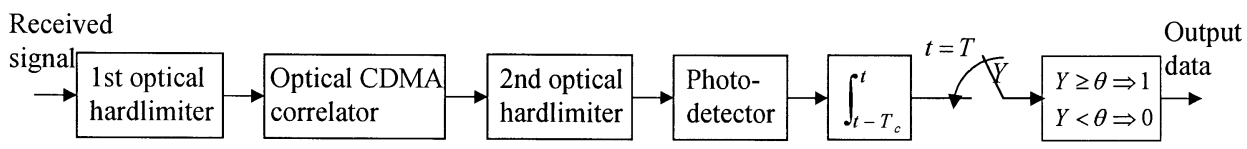

(a)

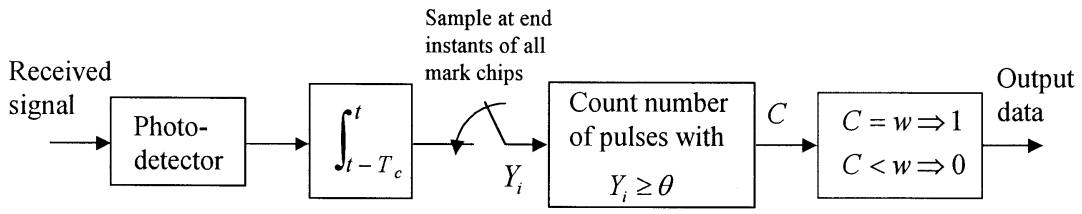

(b)

Fig. 1. Optical OOK-CDMA receiver models. (a) Correlation receiver with double optical hardlimiters. (b) Chip-level receiver.

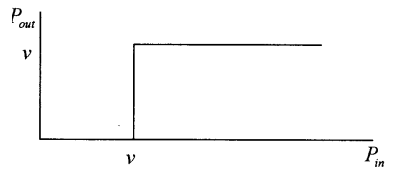

(a)

(c)

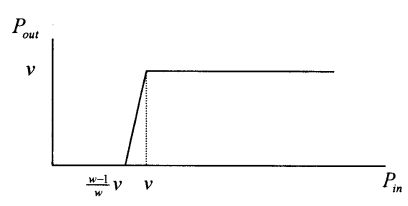

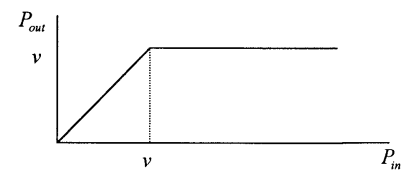

(b)

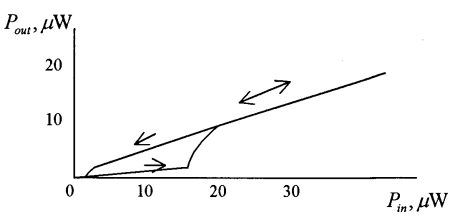

(d)
Fig. 2. Optical hardlimiters' characteristics: (a) an ideal optical hardlimiter; (b) suitable for the first optical hardlimiter; (c) suitable for the second optical hardlimiter; (d) a practical optical hardlimiter.

\section{OPTICAL OOK-CDMA RECEIVER MODELS AND HARDWARE COMPLEXITY}

The block diagram of the double-optical-hardlimiters correlation receiver is shown in Fig. 1(a), where $T$ and $T_{c}$ are the bit time and the chip time durations, respectively. The output function of the ideal optical hardlimiter is defined as

$$
g(x)= \begin{cases}u, & \text { if } x \geq v \\ 0, & \text { otherwise }\end{cases}
$$

where $x$ denotes the input optical power, $v$ denotes the threshold power level of the optical hardlimiter, and $u$ is a constant. Thus, the optical hardlimiter clips the received optical power to $u$ whenever it exceeds the threshold level $v$. In optical CDMA detection, $u$ is usually set equal to $v$. Fig. 2(a) shows the input-output characteristic of an ideal optical hardlimiter, which is impossible to realize in practice. However, when optical hardlimiters are used in the receiver of Fig. 1(a), it is sufficient to implement the characteristic in Fig. 2(b) for the first optical hardlimiter and that in Fig. 2(c) for the second optical hardlimiter. Fig. 2(d) shows the characteristic of a practical hardlimiter, which is distinguished by power loss and two different threshold levels for the set and reset states. Further, the output power remains dependent on the input power after switching. It can be noticed from Fig. 1(a) that three threshold settings are needed for the double-hardlimiters correlation receiver; two for the optical hardlimiters, and one for the OOK decoder. These thresholds are generally dependent on the received optical power and the number of simultaneous users, hence, it is required to dynamically provide information about these parameters. Further, optical hardlimiters with variable thresholds do not exist in practice. The optical CDMA correlator in Fig. 1(a) usually splits the received optical signal into a number of branches, which is equal to the code weight $w$, and then combines these branches after properly delaying the split optical pulses in accordance with the signature code. This splitting process wastes most of the received optical signal, namely, $(w-1) / w$ of the received power is lost. The electronic switch in Fig. 1(a) samples at a rate that is equal to the data bit rate $R_{b}=1 / T$. This rate is much less than the optical processing rate (or chip rate $R_{c}=1 / T_{c}$ ). In fact, $R_{b}=R_{c} / L$, where $L$ is the code length. On the other hand, the integration time after the photodetector is $T_{c}$, which means that very high-speed electronics (at chip rate) are required for this receiver. Recently, Zahedi and Salehi [10] have demonstrated an equivalent (but even more complex) structure that uses an optical active multiplier to extend the integration time to $T$, rather than $T_{c}$.

The block diagram of the chip-level receiver is shown in Fig. 1(b). From this figure, we can see that the chip-level receiver does not require the optical correlator and, hence, it does not waste the received optical power as in the correlation receivers. The information about the signature code is provided in the electronic switch, which samples only at the end instants of the mark chips. The average sampling rate of this electronic switch is still very low compared to the optical processing rate. In fact, it samples at an average rate of $w R_{b}$. Further, the chip-level receiver does not involve optical hardlimiters, and only one threshold is required for the decision system. It turned out that for a shot-noise-limited system, this threshold is even independent of the system parameters, whereas for a more general system, this threshold depends on the received optical power. Again, although the integration time is limited to $T_{c}$, it can be extended to $T$ by the use of active optical multipliers [7], [10]. In this system, only bit-rate operations are performed electronically.

From the above discussion, it turns out that the chip-level receiver is much more practical than the double-hardlimiters correlator. In the next section, we compare their performances. 


\section{BIT ERROR PROBABILITIES FOR OOK-CDMA SYSTEMS WITH $\lambda=1$}

In this section, we compare the performances [in terms of the bit error rates (BERs)] of both chip-level and double-hardlimiters correlation receivers. We consider two cases: 1) Poisson shot-noise-limited photodetectors; and 2) APDs with thermal noise. In our evaluation, we consider only ideal optical hardlimiters for the correlation receivers.

\section{A. Poisson Shot-Noise-Limited Photodetectors}

The bit error probability for the double-hardlimiters correlation receiver with Poisson photodetectors can be found in [6], and that for the chip-level receiver can be found in [7]. That for the latter is restated here for convenience

$$
P_{b}=\frac{1}{2}(P[E \mid 0]+P[E \mid 1])
$$

where

$$
P[E \mid 0]=\sum_{i=0}^{w}(-1)^{i}\left(\begin{array}{c}
w \\
i
\end{array}\right)\left[1-i \frac{w}{2 L}+i \frac{w}{2 L} e^{-Q}\right]^{N-1}
$$

and

$$
P[E \mid 1]=-\sum_{i=1}^{w}(-1)^{i}\left(\begin{array}{c}
w \\
i
\end{array}\right) e^{-Q i}\left[1-i \frac{w}{2 L}+i \frac{w}{2 L} e^{-Q}\right]^{N-1} .
$$

Here, $N$ denotes the number of simultaneous users and $Q$ denotes the average photons per chip pulse. It is related to the average photons per bit $\mu$ as follows:

$$
Q= \begin{cases}\frac{2 \mu}{w}, & \text { for chip-level receivers } \\ \frac{2 \mu}{w^{2}}, & \text { for correlation receivers. }\end{cases}
$$

In the limiting case, when $Q \rightarrow \infty$, the last two probabilities reduce to

$$
P_{\infty}[E \mid 0]=\sum_{i=0}^{w}(-1)^{i}\left(\begin{array}{c}
w \\
i
\end{array}\right)\left[1-i \frac{w}{2 L}\right]^{N-1}
$$

and

$$
P_{\infty}[E \mid 1]=0
$$

respectively. It should be emphasized that the error probability for the chip-level receiver from [7] was derived under the assumption of a constant threshold $\theta=1$ [cf. Fig. 1(b)]. This threshold is independent of the number of users and the average optical power, which adds to the advantages of chip-level receivers.

The error probabilities for both receivers are plotted in Fig. 3, versus the average received photons per bit, for different system parameters. An optimum threshold has been used for the double-hardlimiters correlation receiver, whereas a suboptimum threshold $\theta=1$ has been used for the chip-level receiver. From this figure, we can see that the BER of the double-hardlimiters correlation receiver is slightly better than that of the chip-level receiver for very low optical power. Soon they coincide with each other (by increasing the average optical power) and reach the probability error floor. It

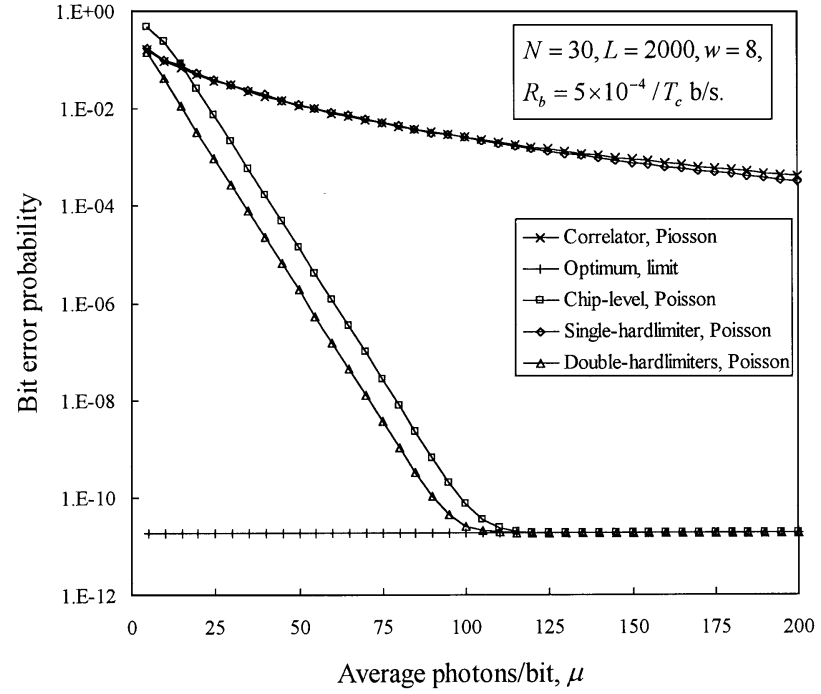

Fig. 3. Bit error probabilities for OOK-CDMA receivers, under a Poisson shot-noise-limited assumption, versus average photons/bit.

should be emphasized that although the performance of the double-hardlimiter correlator is slightly better, it is expected to be worse than that of the chip-level receiver in practice, since the properties of the ideal sharp hardlimiter are impossible to practically realize. The error probabilities for the optimum receiver, and correlation receivers without hardlimiters and with a single hardlimiter, are also plotted in the same figure for convenience.

\section{B. Avalanche Photodetectors and Thermal Noise}

The bit error probability for the double-hardlimiters correlation receiver when using an APD, and taking into account the effect of the thermal noise, can be found in [8]. We derive here the corresponding BER, $P_{b}$, for the chip-level receiver. $P_{b}$ can be obtained as in (2) with

$$
\begin{array}{r}
P[E \mid b]=\sum_{l_{1}, l_{2}, \ldots, l_{w}} \operatorname{Pr}\left\{\kappa_{1}=l_{1}, \kappa_{2}=l_{2}, \ldots, \kappa_{w}=l_{w}\right\} \\
\times P\left[E \mid D=b, \kappa_{1}=l_{1}, \ldots, \kappa_{w}=l_{w}\right]
\end{array}
$$

where $P\left[E \mid D=b, \kappa_{1}=l_{1}, \ldots, \kappa_{w}=l_{w}\right]$ denotes the probability of error, given data bit $b \in\{0,1\}$ and an interference pattern $\left\{\kappa_{1}=l_{1}, \kappa_{2}=l_{2}, \ldots, \kappa_{w}=l_{w}\right\}$. Here, $\kappa_{i}$, $i \in \mathcal{X} \stackrel{\text { def }}{=}\{1,2, \ldots, w\}$, denotes the number of pulses from other users that interfere to chip $i$ of the mark positions of the signature code of the desired user. It was shown in [7] that the random vector $\left(\kappa_{1}, \kappa_{2}, \ldots, \kappa_{w}\right)^{T}$ admits a multinomial distribution with parameters $N-1$ and $w / 2 L$

$$
\begin{gathered}
\operatorname{Pr}\left\{\kappa_{1}=l_{1}, \ldots, \kappa_{w}=l_{w}\right\}=\frac{(N-1) !}{l_{1} ! l_{2} ! \cdots l_{w} !\left(N-1-S_{w}\right) !} \\
\cdot\left(\frac{w}{2 L}\right)^{S_{w}}\left(1-\frac{w^{2}}{2 L}\right)^{N-1-S_{w}}
\end{gathered}
$$

where

$$
S_{w}=\sum_{i=1}^{w} l_{i} .
$$


Denoting by $Y_{i}, i \in \mathcal{X}$, the number of photons collected from chip $i$ of the mark positions of the signature code of the desired user [cf. Fig. 1(b)], we have

$$
\begin{aligned}
P[E \mid D & \left.=1, \kappa_{1}=l_{1}, \ldots, \kappa_{w}=l_{w}\right] \\
& =\operatorname{Pr}\left\{Y_{i}<\theta, \text { some } i \in \mathcal{X} \mid D=1\right. \\
\left.\kappa_{1}=l_{1}, \ldots, \kappa_{w}=l_{w}\right\} & \left.\kappa_{1}=l_{1}, \ldots, \kappa_{w}=l_{w}\right\} \\
& =-\sum_{i=1}^{w}(-1)^{i}\left(\begin{array}{c}
w \\
i
\end{array}\right) \operatorname{Pr}\left\{Y_{j}<\theta \quad \forall j \leq i \mid D=1\right. \\
& =-\sum_{i=1}^{w}(-1)^{i}\left(\begin{array}{c}
w \\
i
\end{array}\right) \prod_{j=1}^{i} \operatorname{Pr}\left\{Y_{j}<\theta \mid D=1, \kappa_{j}=l_{j}\right\} \\
& =-\sum_{i=1}^{w}(-1)^{i}\left(\begin{array}{c}
w \\
i
\end{array}\right) \prod_{j=1}^{i} Q\left(\frac{m_{1 j}-\theta}{\sigma_{1 j}}\right)
\end{aligned}
$$

and similarly

$$
\begin{aligned}
P[E \mid D= & \left.0, \kappa_{1}=l_{1}, \ldots, \kappa_{w}=l_{w}\right] \\
= & \operatorname{Pr}\left\{Y_{i} \geq \theta, \forall i \in \mathcal{X} \mid D=0\right. \\
& \left.\kappa_{1}=l_{1}, \ldots, \kappa_{w}=l_{w}\right\} \\
= & 1-\operatorname{Pr}\left\{Y_{i}<\theta, \text { some } i \in \mathcal{X} \mid D=0\right. \\
& \left.\kappa_{1}=l_{1}, \ldots, \kappa_{w}=l_{w}\right\} \\
= & 1+\sum_{i=1}^{w}(-1)^{i}\left(\begin{array}{c}
w \\
i
\end{array}\right) \prod_{j=1}^{i} Q\left(\frac{m_{0 j}-\theta}{\sigma_{0 j}}\right)
\end{aligned}
$$

where the function $Q(x)$ is the normalized Gaussian tail probability

$$
Q(x)=\frac{1}{\sqrt{2 \pi}} \int_{x}^{\infty} e^{-s^{2} / 2} d s
$$

$m_{b j}$ and $\sigma_{b j}^{2}$ are the conditional mean and variance, respectively, of the decision variable $Y_{j}, j \in \mathcal{X}$, given data bit $b \in\{0,1\}$ and interference pattern $\left\{\kappa_{1}=l_{1}, \kappa_{2}=l_{2}, \ldots, \kappa_{w}=l_{w}\right\}$. They are given by

$$
m_{b j}=G\left(q b+q l_{j}+q_{d}\right), \quad \sigma_{b j}^{2}=F G m_{b j}+\sigma_{n}^{2} .
$$

Here, $G$ is the average APD gain, $q$ is the average number of absorbed photons per received single-user pulse, $q_{d}$ is the photon count due to the APD dark current within a chip interval, and $\sigma_{n}^{2}$ is the variance of the thermal noise within a chip interval. $q$, $q_{d}$, and $\sigma_{n}^{2}$ are given by

$$
q=\frac{\eta \lambda_{o} P_{\mathrm{av}} T}{h c w}, \quad q_{d}=\frac{I_{d} T_{c}}{e}, \quad \sigma_{n}^{2}=\frac{2 k_{B} T^{\circ}}{e^{2} R_{L}} T_{c}
$$

respectively, where $P_{\text {av }}$ is the average received laser power (of a single user), $\lambda_{o}$ is the laser wavelength, $\eta$ is the APD efficiency, $h=6.626 \times 10^{-34} \mathrm{~J} . \mathrm{s}$ is Plank's constant, $c=3 \times 10^{8} \mathrm{~m} / \mathrm{s}$ is the speed of light, $I_{d}$ is the APD dark current, $e=1.6 \times 10^{-19} \mathrm{C}$ is the electron charge, $k_{B}=1.38 \times 10^{-23} \mathrm{~J} / \mathrm{K}$ is Boltzmann's constant, $T^{\circ}$ is the receiver noise temperature, and $R_{L}$ is the

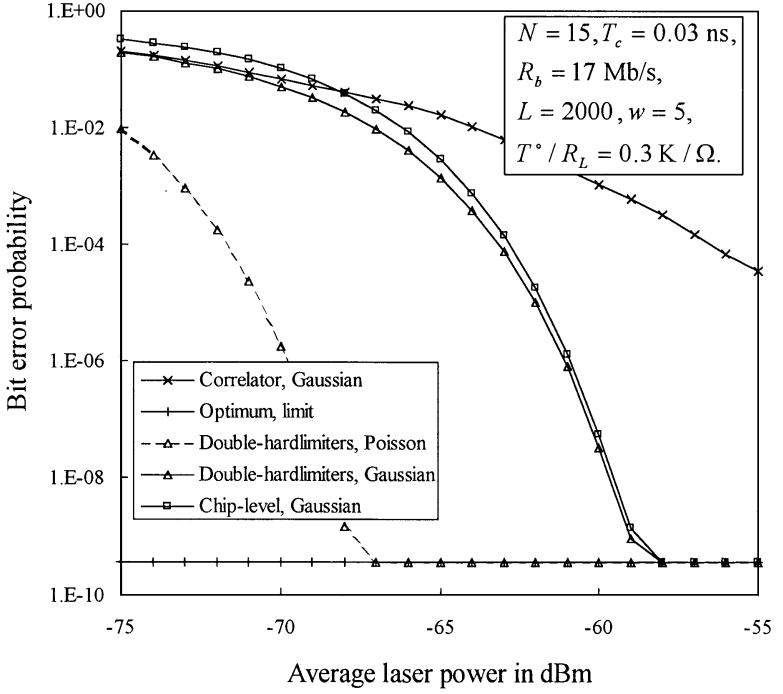

Fig. 4. Bit error probabilities for OOK-CDMA receivers, with APD noise and low thermal noise, versus the average laser power.

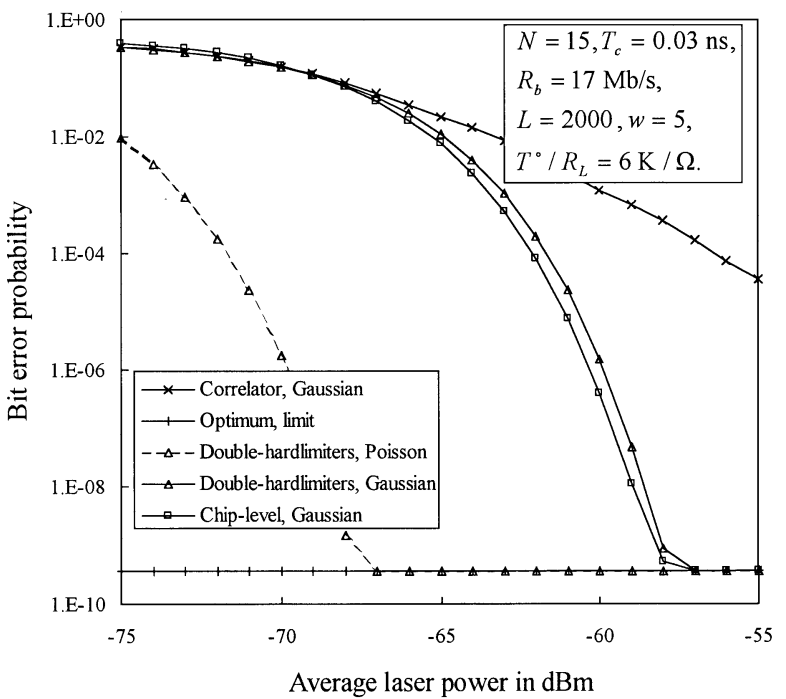

Fig. 5. Bit error probabilities for OOK-CDMA receivers, with APD noise and high thermal noise, versus the average laser power.

receiver load resistor. $F$ is the APD excess noise factor, given by

$$
F=k_{\mathrm{eff}} G+(2-1 / G)\left(1-k_{\mathrm{eff}}\right)
$$

where $k_{\text {eff }}$ is the APD effective ionization ratio. Substituting (10) and (11) in (7) and (2), we get

$$
\begin{aligned}
P_{b}= & \frac{1}{2}+\frac{1}{2} \sum_{l_{1}, l_{2}, \ldots, l_{w}} \operatorname{Pr}\left\{\kappa_{1}=l_{1}, \kappa_{2}=l_{2}, \ldots, \kappa_{w}=l_{w}\right\} \\
& \times \sum_{i=1}^{w}(-1)^{i}\left(\begin{array}{c}
w \\
i
\end{array}\right) \\
& \times\left[\prod_{j=1}^{i} Q\left(\frac{m_{0 j}-\theta}{\sigma_{0 j}}\right)-\prod_{j=1}^{i} Q\left(\frac{m_{1 j}-\theta}{\sigma_{1 j}}\right)\right] .
\end{aligned}
$$

The error probabilities for both receivers in this case are plotted in Figs. 4 and 5, versus the average received laser 


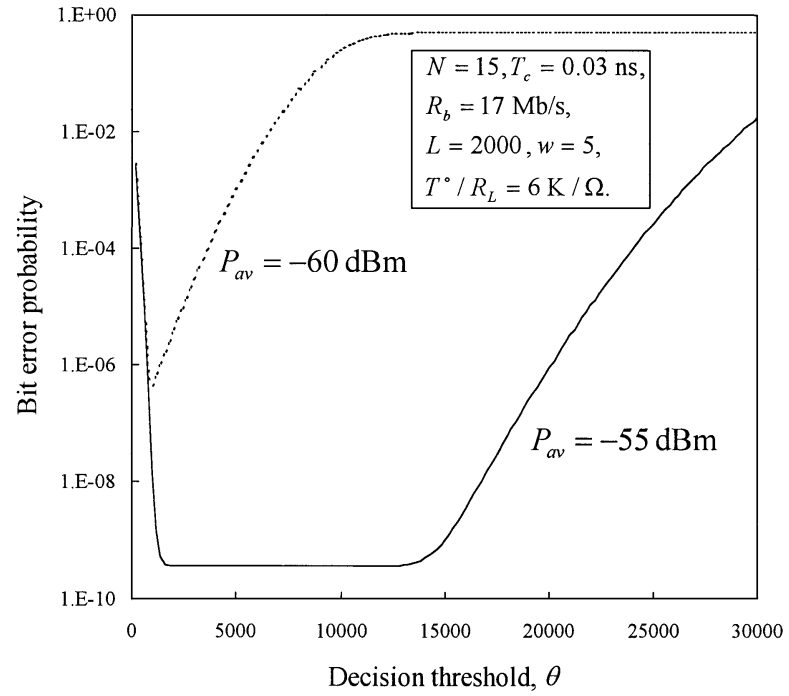

Fig. 6. Bit error probabilities for OOK-CDMA chip-level receivers versus the decision threshold.

power, with different system parameters. Optimum thresholds have been used for both receivers. The error probabilities for the optimum receiver, correlation receiver without hardlimiters, and Poisson shot-noise-limited double-hardlimiters correlation receiver are also plotted in the same figure for convenience. The following system parameters are used in our calculations. The laser pulsewidth $T_{c}=0.03 \mathrm{~ns}$, the average APD gain $G=100$, its unit gain responsivity $\mathcal{R}=e \eta \lambda_{o} / h c=0.84 \mathrm{~A} / \mathrm{W}$, its effective ionization ratio $k_{\text {eff }}=0.02$, its dark current $I_{d}=1 \mathrm{nA}$, and the receiver normalized noise temperature $T^{\circ} / R_{L} \in\{0.3,6\} \mathrm{K} / \Omega$. From Figs. 4 and 5 , we can see that the chip-level receiver performs slightly better than the double-hardlimiter correlation receiver when the thermal noise is high, whereas the double-hardlimiter correlation receiver performs slightly better than the chip-level receiver for low thermal noise.

In Fig. 6, we plot the probability of error of chip-level receivers for two different values of the average received optical power, versus the decision threshold $\theta$. It can be seen that when the optical power is large enough (so that the error probability floor is reached), the optimum threshold is not unique, and it covers a practically wide range. However, when the optical power is not that large, then there is only one unique optimum threshold. In practice, the error probability floor can be easily achieved even with sufficiently low laser power (cf. Figs. 4 and 5).

\section{BIT ERror PRobAbILITIES FOR OOK-CDMA SySTEMS WITH $\lambda=2$}

\section{A. Interference Probability}

Let $p_{t}, t \in\{1,2\}$, denote the probability that a single user interferes with the desired user at $t$ mark positions. It was shown in [3] that

$$
p_{1}+2 p_{2}=\frac{w^{2}}{2 L}
$$

In this section, we only study the worst case, which occurs when $p_{1}=0$ in the last equation, and hence

$$
p_{2}=\frac{w^{2}}{4 L}
$$

That is, if a single user interferes with the desired user, it will cause interference to exactly two mark positions of the desired user. The system performance, in this case, provides an upper bound to the more general one with $p_{1} \geq 0$ [3].

As was mentioned earlier, we denote by $\kappa_{i}, i \in \mathcal{X}=$ $\{1,2, \ldots, w\}$, the number of pulses (from other users) that interfere to chip $i$ of the mark positions of the signature code of the desired user. Further, we denote by $n_{i j}, j>i$, and $i, j \in \mathcal{X}$ the number of users that interfere with both chips $i$ and $j$ of the mark positions of the desired user. Thus, we have the following set of linear equations [3]:

$$
\begin{aligned}
\kappa_{1} & =n_{12}+n_{13}+\cdots+n_{1 w} \\
\kappa_{2} & =n_{12}+n_{23}+\cdots+n_{2 w} \\
& \vdots \\
\kappa_{w} & =n_{1 w}+n_{2 w}+\cdots+n_{w-1, w}
\end{aligned}
$$

To find the probability distribution of the random variables $n_{i j}$, we proceed as follows. Let $A_{i j}, j>i$, and $i, j \in \mathcal{X}$ be the event that a single user interferes with the desired user at chips $i$ and $j$ of its mark positions. The events $\left\{A_{i j}: j>i\right.$ and $i, j \in \mathcal{X}\}$ are disjoint and their total count is $\left(\begin{array}{c}w \\ 2\end{array}\right)$. Assuming uniform delays among all users, we calculate the probability of each of these events as follows. Denote the probability of $A_{i j}$ by $\rho$

$$
\begin{aligned}
\rho & =\operatorname{Pr}\left\{A_{i j}\right\}=\operatorname{Pr}\left\{n_{i j}=1 \mid N=2\right\} \\
& =\operatorname{Pr}\left\{\kappa_{i}=\kappa_{j}=1 \mid N=2\right\} \\
& =\operatorname{Pr}\left\{\kappa_{i}=1 \mid N=2\right\} \cdot \operatorname{Pr}\left\{\kappa_{j}=1 \mid N=2, \kappa_{i}=1\right\} \\
& =\frac{w}{2 L} \cdot \frac{1}{w-1}=\frac{w}{2 L(w-1)} .
\end{aligned}
$$

Further, let $A_{0}$ be the event that a single user does not interfere at all with the desired user at any of its $w$ mark positions. Thus

$$
\operatorname{Pr}\left\{A_{0}\right\}=1-\left(\begin{array}{l}
w \\
2
\end{array}\right) \rho=1-\frac{w^{2}}{4 L}
$$

It is obvious from the above discussion that the random variables $\left\{n_{i j}: j>i\right.$ and $\left.i, j \in \mathcal{X}\right\}$ admit a multinomial distribution with parameters $N-1$ and $\rho$

$$
\begin{aligned}
\operatorname{Pr} & \left\{\mathbf{n}_{1}=\mathbf{l}_{1}, \quad \mathbf{n}_{2}=\mathbf{l}_{2}, \ldots, \mathbf{n}_{w-1}=1_{w-1}\right\} \\
= & \frac{(N-1) !}{(N-1-S) ! \cdot \prod_{i=1}^{w-1}} \prod_{j=i+1}^{w} l_{i j} !
\end{aligned}
$$


where, for every $i \in\{1,2, \ldots, w-1\}, \mathbf{n}_{i}$ and $1_{i}$ denote the vectors $\left(n_{i, i+1}, n_{i, i+2}, \ldots, n_{i, w}\right)^{T}$ and $\left(l_{i, i+1}, l_{i, i+2}, \ldots, l_{i, w}\right)^{T}$, respectively, and

$$
S=\sum_{i=1}^{w-1} \sum_{j=i+1}^{w} l_{i j}
$$

As was denoted earlier, we let $Y_{i}, i \in \mathcal{X}$, be the photon count collected from chip number $i$ of the mark positions of the signature code of the desired user. In order to simplify the analysis and have some insights on the problem under consideration, we focus here on the Poisson shot-noise-limited case only.

\section{B. Decision Rule}

If the collected photon count from each mark chip of the underlying code is positive, " 1 " is declared, otherwise " 0 " is declared to be sent. That is

$$
\text { Decide } \begin{cases}1, & \text { if } Y_{i} \geq 1 \quad \forall i \in \mathcal{X} \\ 0, & \text { otherwise. }\end{cases}
$$

\section{Error Probability for Chip-Level Receivers}

The probability of bit error is, thus, given by (2) with

$$
\begin{aligned}
P[E \mid 1]= & \operatorname{Pr}\left\{Y_{i}=0, \text { some } i \in \mathcal{X} \mid D=1\right\} \\
= & -\sum_{i=1}^{w}(-1)^{i}\left(\begin{array}{c}
w \\
i
\end{array}\right) \\
& \times \operatorname{Pr}\left\{Y_{1}=Y_{2}=\cdots=Y_{i}=0 \mid D=1\right\}
\end{aligned}
$$

and

$$
\begin{aligned}
P[E \mid 0]= & \operatorname{Pr}\left\{Y_{i} \geq 1 \quad \forall i \in \mathcal{X} \mid D=0\right\} \\
= & 1-\operatorname{Pr}\left\{Y_{i}=0, \text { some } i \in \mathcal{X} \mid D=0\right\} \\
= & 1+\sum_{i=1}^{w}(-1)^{i}\left(\begin{array}{c}
w \\
i
\end{array}\right) \\
& \times \operatorname{Pr}\left\{Y_{1}=Y_{2}=\cdots=Y_{i}=0 \mid D=0\right\} .
\end{aligned}
$$

Thus, for $b \in\{0,1\}$, we can evaluate the probabilities under the last two summations as follows:

$$
\begin{aligned}
\operatorname{Pr} & \left\{Y_{1}=Y_{2}=\cdots=Y_{i}=0 \mid D=b\right\} \\
= & \sum_{l_{1}, \ldots, l_{i}} \operatorname{Pr}\left\{Y_{1}=Y_{2}=\cdots=Y_{i}=0 \mid D=b,\right. \\
& \left.\kappa_{1}=l_{1}, \ldots, \kappa_{i}=l_{i}\right\} \times \operatorname{Pr}\left\{\kappa_{1}=l_{1}, \ldots, \kappa_{i}=l_{i}\right\} \\
= & \sum_{l_{1}, \ldots, l_{i}} \operatorname{Pr}\left\{\kappa_{1}=l_{1}, \ldots, \kappa_{i}=l_{i}\right\} \\
& \times \prod_{j=1}^{i} \operatorname{Pr}\left\{Y_{j}=0 \mid D=b, \kappa_{j}=l_{j}\right\} \\
= & \sum_{l_{1}, \ldots, l_{i}} \operatorname{Pr}\left\{\kappa_{1}=l_{1}, \ldots, \kappa_{i}=l_{i}\right\} \prod_{j=1}^{i} \exp \left[-Q\left(b+l_{j}\right)\right] \\
= & \exp [-Q b i] \cdot E\left\{\exp \left[-Q \sum_{j=1}^{i} \kappa_{j}\right]\right\}
\end{aligned}
$$

Here, the second equality holds because the random variables $Y_{1}, \ldots, Y_{i}$ are independent given $\kappa_{1}, \ldots, \kappa_{i}$, and $E$ denotes the expected value. To evaluate the last expectation, we notice from (19) that

$$
\sum_{j=1}^{i} \kappa_{j}=2 \sum_{r=1}^{i-1} \sum_{s=r+1}^{i} n_{r s}+\sum_{r=1}^{i} \sum_{s=i+1}^{w} n_{r s}=2 \alpha+\beta
$$

where

$$
\alpha=\sum_{r=1}^{i-1} \sum_{s=r+1}^{i} n_{r s} \text { and } \beta=\sum_{r=1}^{i} \sum_{s=i+1}^{w} n_{r s} .
$$

From (22) and (29), it turns out that the vector $(\alpha, \beta)^{T}$ admits a trinomial distribution with parameters $N-1, i(i-1) \rho / 2$, and $i(w-i) \rho$. Thus

$$
\begin{aligned}
& E\left\{\exp \left[-Q \sum_{j=1}^{i} \kappa_{j}\right]\right\} \\
& =E\{\exp [-Q(2 \alpha+\beta)]\} \\
& =\left[1-i \frac{i-1}{2} \rho-i(w-i) \rho+i \frac{i-1}{2} \rho e^{-2 Q}\right. \\
& \left.\quad+i(w-i) \rho e^{-Q}\right]^{N-1} \\
& =\left[1-i\left(w-1-\frac{i-1}{2}\right) \rho+i \frac{i-1}{2} \rho e^{-2 Q}\right. \\
& \left.\quad+i(w-i) \rho e^{-Q}\right]^{N-1} .
\end{aligned}
$$

By substitution in (27) and then in both (26) and (25), we obtain

$$
\begin{gathered}
P[E \mid 0]=\sum_{i=0}^{w}(-1)^{i}\left(\begin{array}{c}
w \\
i
\end{array}\right) \times\left[1-i\left(w-1-\frac{i-1}{2}\right) \rho\right. \\
\left.+i \frac{i-1}{2} \rho e^{-2 Q}+i(w-i) \rho e^{-Q}\right]^{N-1}
\end{gathered}
$$

and

$$
\begin{gathered}
P[E \mid 1]=-\sum_{i=1}^{w}(-1)^{i}\left(\begin{array}{c}
w \\
i
\end{array}\right) e^{-Q i} \times\left[1-i\left(w-1-\frac{i-1}{2}\right) \rho\right. \\
\left.+i \frac{i-1}{2} \rho e^{-2 Q}+i(w-i) \rho e^{-Q}\right]^{N-1}
\end{gathered}
$$

respectively. In the limiting case, when $Q \rightarrow \infty$, the last two probabilities reduce to

$$
\begin{aligned}
& P_{\infty}[E \mid 0]=\sum_{i=0}^{w}(-1)^{i}\left(\begin{array}{c}
w \\
i
\end{array}\right)\left[1-i \frac{w}{4 L}\left(2-\frac{i-1}{w-1}\right)\right]^{N-1} \\
& \text { and } \\
& P_{\infty}[E \mid 1]=0
\end{aligned}
$$

respectively.

Equations (2), (31), and (32) provide the error probability calculations for chip-level receivers under a Poisson shot-noiselimited assumption. The corresponding error rates for the correlation receivers without optical hardlimiters are found in [3], but only with ideal photodetectors, i.e., when $Q \rightarrow \infty$. 


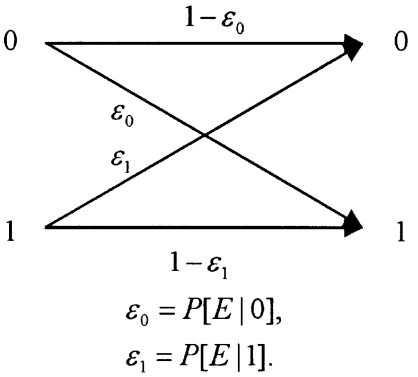

(a)

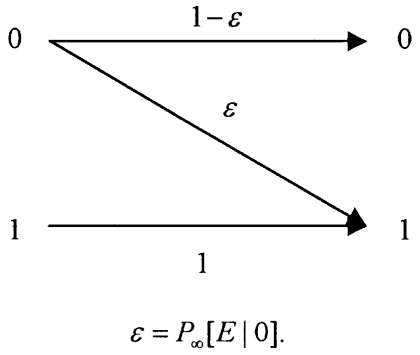

(b)

Fig. 7. Channel models of the optical OOK-CDMA systems. (a) Poisson shot-noise-limited case. (b) Noiseless case.

\section{CAPACITY COMPARISON}

In this section, we compare between the throughput capacities of the OOK-CDMA systems with $\lambda \in\{1,2\}$. We consider here both chip-level receivers and correlation receivers without hardlimiters.

\section{A. Channel Models}

We assume, in our channel models, that the desired receiver is only interested in the message transmitted by the desired transmitter, i.e., no cooperation between users is permitted. Thus, the multiple-access channel is reduced to $N$ single-user channels [15], and each is subject to multiple-user interference from the others. In our evaluation of the channel capacities, we calculate the mutual information $I(X \wedge Y)$ between the binary input $X=\{0,1\}$ and the binary output $Y=\{0,1\}$, for equiprobable inputs. Of course, this yields lower bounds to the actual channel capacities, but is suited to most practical coding schemes [14]. Further, the corresponding computation is slightly simpler. The channel model is shown in Fig. 7(a), which is a binary asymmetric channel. For sufficiently large values of $Q$, this channel reduces to the $Z$ channel shown in Fig. 7(b).

\section{B. Definitions}

The channel capacity in nats per channel use, the total throughput capacity in nats per second, and the efficiency capacity in nats per photon are defined as

$$
\begin{aligned}
C & =I(X \wedge Y) \quad \text { nats } / \mathrm{cu} \\
C_{T} & =\frac{N C}{T}=\frac{N C}{L T_{c}} \quad \text { nats } / \mathrm{s} \\
C_{p h} & =\frac{C}{\mu} \quad \text { nats/ph. }
\end{aligned}
$$

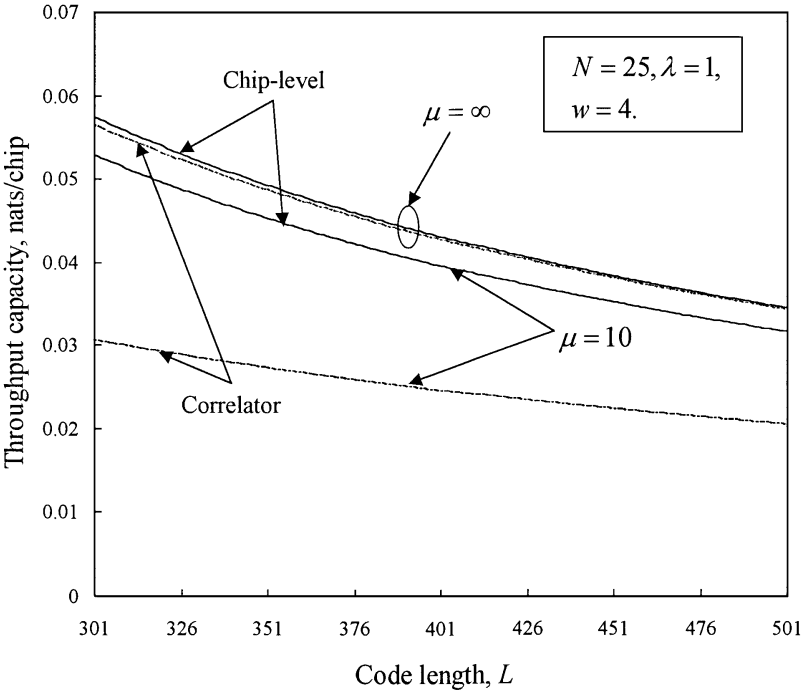

Fig. 8. Throughput capacities versus the code length for both chip-level and correlation OOK-CDMA systems when $N=25, \lambda=1$, and $w=4$.

It is easy to check that the mutual information functions for the channels of Fig. 7 (in the case of equiprobable inputs) are given by

$$
\begin{aligned}
& I(X \wedge Y) \\
& \quad= \begin{cases}\log 2-\frac{1-\epsilon_{1}+\epsilon_{0}}{2} h\left(\frac{\epsilon_{0}}{1-\epsilon_{1}+\epsilon_{0}}\right) & \text { Poisson case } \\
-\frac{1-\epsilon_{0}+\epsilon_{1}}{2} h\left(\frac{\epsilon_{1}}{1-\epsilon_{0}+\epsilon_{1}}\right) & \text { ideal case }\end{cases}
\end{aligned}
$$

where

$$
\epsilon_{0}=P[E \mid 0], \quad \epsilon_{1}=P[E \mid 1], \quad \epsilon=P_{\infty}[E \mid 0]
$$

and $h(p)$ is the binary entropy function, given by

$$
h(p)=-p \log p-(1-p) \log (1-p) .
$$

\section{Numerical Results}

The throughput capacities of both correlation and chip-level OOK-CDMA systems with $\lambda=1$ are shown in Fig. 8 versus the code length when $N=25, w=4$, and $\mu=10$. In view of the OOC constraint [1]

$$
N \leq \frac{(L-1)(L-2) \cdots(L-\lambda)}{w(w-1) \cdots(w-\lambda)}
$$

the code length should be greater than $1+w(w-1) N=301$ when $\lambda=1$. The advantage of chip-level receivers over that of correlation receivers is obvious from the figure. The limiting throughput capacities when $\mu \rightarrow \infty$ for both systems are depicted in the same figure as well. It can be seen that the increase in the chip-level system's capacity is not that significant when $\mu$ increases above ten, whereas, that of the correlation 


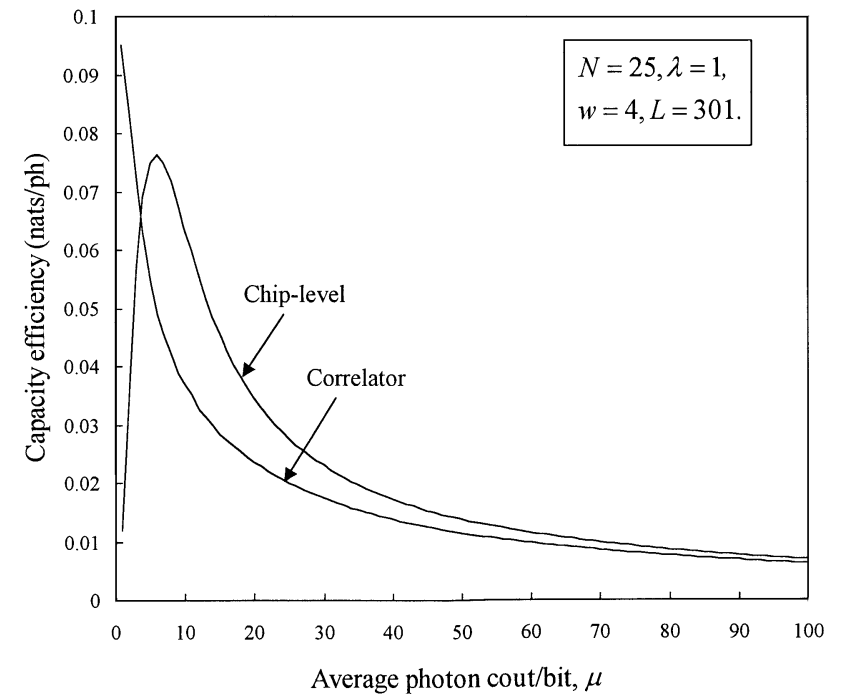

Fig. 9. Capacity efficiencies versus the average photons per bit for both chip-level and correlation OOK-CDMA systems when $N=25, \lambda=1$, $L=301$, and $w=4$.

system is very large. This demonstrates that chip-level receivers are very efficient in utilizing the optical power. The maximum throughput is achieved at the boundary of the feasible region, i.e., at minimum $L$.

The capacity efficiencies are plotted in Fig. 9 for $\lambda=1$ and minimum value of $L$. For the case of the correlation receiver, the efficiency decreases as the average number of photons $\mu$ increases, whereas, there exists an optimum value of $\mu$ that maximizes the efficiency for the case of chip-level receiver. This is because we did not choose an optimum threshold for chip-level receivers, which makes the system a suboptimum one for very low values of $\mu$. However, when $\mu$ increases a bit, the threshold becomes optimum, and chip-level receivers become more efficient. It is also seen from both Figs. 8 and 9 that for large values of $\mu$, the capacities of both systems coincide and the advantages of the chip-level receivers are lost.

From Fig. 8, it can be seen that the throughput can be increased by decreasing $L$ below the boundary of the feasible region. To mine this advantage, we can widen the feasible region by switching $\lambda$ to 2 , which increases the possible values of the code length, that is, $L \geq 26$ for $N=25$ and $w=4$. The corresponding capacities are plotted in Fig. 10 for $\mu=\infty$. It is obvious that the optimum values of the code lengths occur inside the feasible region and the advantages of chip-level receivers are retained even for infinite power. The throughput capacity when $\mu=10$ for chip-level systems is depicted in the same figure as well. It can be seen that it is very close to the limiting throughput capacity, which confirms the efficiency of chip-level receivers even for $\lambda=2$. Comparing Fig. 8 with Fig. 10, we conclude that the throughput capacity of chip-level receivers increases by a factor of 3.4 when using $\lambda=2$ rather than $\lambda=1$.

\section{CONCLUSION}

The hardware complexity of both double-optical-hardlimiters correlation receivers and chip-level receivers that are used in recovering optical CDMA signals has been studied and compared.

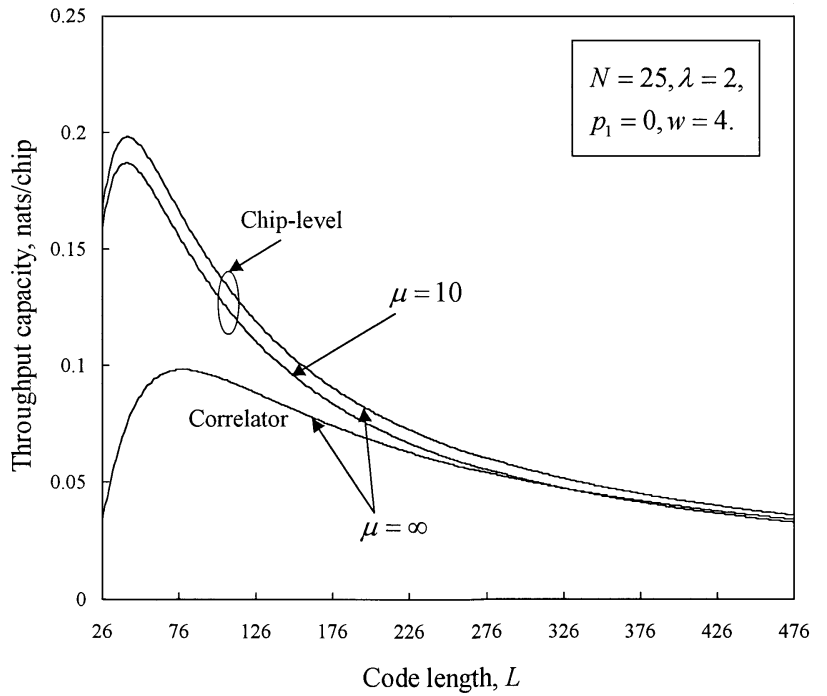

Fig. 10. Throughput capacities versus the code length for both chip-level and correlation OOK-CDMA systems when $N=25, \lambda=2$, and $w=4$.

The bit error probabilities for the latter have been derived under code correlations bounded by one and two, and taking into account the effect of multiple-user interference. For the case of a code-correlation constraint of two, only the Poisson shot-noiselimited case has been considered, whereas, for the case of a code-correlation constraint of one, the effect of both APD noise and thermal noise are considered, in addition to the Poisson shot-noise-limited case. Furthermore, both the throughput capacity and the efficiency of chip-level systems and traditional correlation systems are derived and evaluated. The following concluding remarks can be extracted from our results.

i) Chip-level receivers are much simpler and more practical than double-optical-hardlimiters correlation receivers.

ii) The bit error probabilities of both chip-level receivers and double-optical-hardlimiters receivers are almost similar to each other, even under ideal conditions for the optical hard limiters.

iii) The throughput capacity of chip-level systems is much higher than that of correlation systems whenever the optical power is finite.

iv) When the optical power increases without limit and the code-correlation constraint is equal to one, the last advantage will be lost.

v) This advantage, however, is retained (even for infinite optical power) if the code-correlation constraint is equal to two rather than one.

\section{REFERENCES}

[1] F. R. K. Chung, J. A. Salehi, and V. K. Wei, "Optical orthogonal codes: Design, analysis, and applications," IEEE Trans. Inform. Theory, vol. 35, pp. 595-604, May 1989.

[2] J. A. Salehi and C. A. Brackett, "Code-division multiple-access techniques in optical fiber networks-Part II: Systems performance analysis," IEEE Trans. Commun., vol. 37, pp. 834-842, Aug. 1989.

[3] M. Azizog̃lu, J. A. Salehi, and Y. Li, "Optical CDMA via temporal codes," IEEE Trans. Commun., vol. 40, pp. 1162-1170, July 1992.

[4] H. M. Kwon, "Optical orthogonal code-division multiple-access system-Part I: APD noise and thermal noise," IEEE Trans. Commun., vol. 42, pp. 2470-2479, July 1994. 
[5] M. Brandt-Pearce and B. Aazhang, "Performance analysis of single-user and multiuser detectors for optical code-division multiple-access communication systems," IEEE Trans. Commun., vol. 43, pp. 435-444, Feb.-Apr. 1995.

[6] T. Ohtsuki, "Performance analysis of direct-dectection optical asynchronous CDMA systems with double optical hard-limiters," IEEE J. Lightwave Technol., vol. 15, pp. 452-457, Mar. 1997.

[7] H. M. H. Shalaby, "Chip-level detection in optical code-division multiple-access," IEEE J. Lightwave Technol., vol. 16, pp. 1077-1087, June 1998.

[8] T. Ohtsuki, "Direct-detection optical asynchronous CDMA systems with double optical hard-limiters: APD noise and thermal noise," in Proc. IEEE Global Telecommunications Conf. (GLOBECOM '98), Sydney, Australia, Nov. 8-12, 1998, pp. 1616-1621.

[9] H. M. H. Shalaby, "Synchronous fiber-optic CDMA systems with interference estimators," IEEE J. Lightwave Technol., vol. 17, pp. 2268-2275, Nov. 1999.

[10] S. Zahedi and J. A. Salehi, "Analytical comparison of various fiber-optic CDMA receiver structures," IEEE J. Lightwave Technol., vol. 18, pp. 1718-1727, Dec. 2000

[11] T. W. F. Chang and E. H. Sargent, "Optical CDMA using 2-D codes: The optimal single-user detector," IEEE Commun. Lett., vol. 5, pp. 169-171, Apr. 2001

[12] J. L. Chern, K. Otsuka, and P. Mandel, "Effects induced by transit time on a coupled two-element optical bistable device," IEEE J. Quantum Electron., vol. 28, pp. 555-561, Feb. 1992.

[13] G. Hayashi, S. Kodama, Y. Yamaoka, A. Takano, K. Hirakimoto, Y. Lee, and M. Yamanishi, "Charge-induced self-feedback optical bistable device: Switching time and spatial resolution," IEEE J. Quantum Electron., vol. 29, pp. 2655-2662, Dec. 1993.

[14] I. Bar-David and G. Kaplan, "Information rates of photon-limited overlapping pulse position modulation channels," IEEE Trans. Inform. Theory, vol. IT-30, pp. 455-464, May 1984.

[15] J. G. Goh and S. V. Marić, "The capacities of frequency-hopped codedivision multiple-access channels," IEEE Trans. Inform. Theory, vol. 44, pp. 1204-1211, May 1998

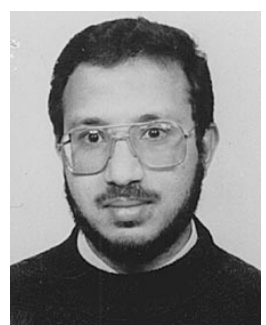

Hossam M. H. Shalaby ( (' 83-M'91-SM'99) was born in Giza, Egypt, in 1961. He received the B.S. and M.S. degrees from the University of Alexandria, Alexandria, Egypt, in 1983 and 1986, respectively, and the Ph.D. degree from the University of Maryland, College Park, in 1991, all in electrical engineering.

In 1991, he joined the Department of Electrical Engineering, University of Alexandria, where he is currently a Full Professor. He has been an Adjunct Professor, Department of Electrical and Information Engineering, Faculty of Sciences and Engineering, Laval University, Quebec, PQ, Canada since January 2001. From March to April 1996, he was a Visiting Professor at the Electrical Engineering Department, Beirut Arab University, Lebanon. From September 1996 to January 1998, he was an Associate Professor with the Electrical and Computer Engineering Department, International Islamic University Malaysia, and from February 1998 to December 1998 , he was with the School of Electrical and Electronic Engineering, Nanyang Technological University, Singapore, where he was a Senior Lecturer, and from January 1999 to February 2001, an Associate Professor. His research interests include optical communications, optical CDMA, spread-spectrum communications, and information theory.

Dr. Shalaby received an SRC fellowship from 1987 to 1991 (Systems Research Center, MD), and both a State Award and Soliman Abd-El-Hay Award in 1995 (Academy of Scientific Research and Technology, Egypt). $\mathrm{He}$ has served as a Student Branch Counselor (Alexandria University), IEEE Alexandria \& North Delta Subsection, since 2002, and served as a Chairman of the Student Activities Committee of IEEE Alexandria Subsection from 1995 to 1996. He has also served as a technical referee for Proceedings of the Institution of Electrical Engineering, IEEE TRANSACTIONS ON COMMUNICATIONS, IEEE TRANSACTIONS ON INFORMATION THEORY, IEEE JOURNAL ON SELECTED AREAS IN COMMUNICATIONS, and the IEEE JOURNAL OF LIGHTWAVE TECHNOLOGY. He is listed in the 14th edition of Marquis Who's Who in the World, 1997. 